Article

\title{
The Effect of Normally Consumed Amounts of Sucrose or High Fructose Corn Syrup on Lipid Profiles, Body Composition and Related Parameters in Overweight/Obese Subjects
}

\section{Joshua Lowndes ${ }^{1}$, Stephanie Sinnett ${ }^{1}$, Sabrina Pardo ${ }^{1}$, Von T. Nguyen ${ }^{1}$, Kathleen J. Melanson ${ }^{2}$,} Zhiping Yu ${ }^{3}$, Britte E. Lowther ${ }^{4}$ and James M. Rippe ${ }^{1,5,6, *}$

1 Rippe Lifestyle Institute, 215 Celebration Place, Suite 300, Celebration, FL 34747, USA;

E-Mails: jlowndes@rippelifestyle.com (J.L.); ssinnett@rippelifestyle.com (S.S.);

sabrinapardo@hotmail.com (S.P.); vnguyen@rippelifestyle.com (V.T.N.)

2 Energy Balance Laboratory, Department of Nutrition and Food Sciences, University of Rhode

Island, Kingston, RI 02881, USA; E-Mail: kmelanson@uri.edu

3 Department of Nutrition and Dietetics, University of North Florida, 1 UNF Drive, Jacksonville, FL 32256, USA; E-Mail: zhiping.nutrition@gmail.com

4 Britte Lowther, 4302 39th Street West, Apt \# 11, Bradenton, FL 34015 , USA;

E-Mail: Britte.lowther@gmail.com

5 Rippe Lifestyle Institute, 21 North Quinsigamond Avenue, Shrewsbury, MA 01545, USA

6 Biomedical Sciences, University of Central Florida, 4000 Central Florida Blvd Orlando, FL 32816, USA

* Author to whom correspondence should be addressed; E-Mail: jrippe@rippelifestyle.com; Tel.: +1-321-939-2394.

Received: 9 January 2014; in revised form: 27 February 2014 / Accepted: 28 February 2014 / Published: 17 March 2014

\begin{abstract}
The American Heart Association (AHA) has advocated that women and men not consume more than 100 and $150 \mathrm{kcal} /$ day, respectively, from added sugars. These levels are currently exceeded by over $90 \%$ of the adult population in the United States. Few data exist on longer-term metabolic effects when sucrose and High Fructose Corn Syrup (HFCS), the principal sources of added dietary sugars, are consumed at levels typical of the general population. Sixty five overweight and obese individuals were placed on a eucaloric (weight stable) diet for 10-weeks, which incorporated sucrose- or HFCS-sweetened, low-fat milk at $10 \%$ or $20 \%$ of calories in a randomized, double-blinded study. All groups responded similarly (interaction $p>0.05$ ). There was no change in body weight in any of the groups over the 10 -week study, or in systolic or diastolic blood pressure. Likewise,
\end{abstract}


there were no changes in total cholesterol, triglycerides, low-density lipoprotein (LDL), or apolipoprotein B (Apo B). We conclude that (1) when consumed as part of a eucaloric diet fructose-when given with glucose (as normally consumed) does not promote weight gain or an atherogenic lipid profile even when consumed at two to four times the level recently recommended by the AHA. (2) There were no differences between HFCS and sucrose on these parameters.

Keywords: sucrose; high fructose corn syrup; body mass

\section{Introduction}

The prevalence of overweight and obesity have increased significantly over the last 30 years in the United States [1-3]. Furthermore, numerous studies have shown that obesity correlates with established risk factors for coronary heart disease (CHD) [4,5]. In this time period consumption of several macronutrients including added fats, flour/cereals and added sugars have increased [6-8]. Some recent reports have suggested a potential association between intake of sugars and adverse effects on cardiovascular health [9]. In particular, some evidence in humans suggests that increased intake of sugars may raise blood pressure [10-12], although the data are inconsistent [13]. Other studies suggest that increased sugars may raise triglyceride levels and lower high-density lipoprotein (HDL) cholesterol [14-16], although once again, the data are inconsistent [17,18]. Studies relating increased consumption of sugar to low-density lipoprotein (LDL) and LDL particle size are also inconsistent [19,20]. Some [21,22], but not all [23,24], studies have also linked higher consumption of added sugar to increased oxidative stress and inflammation. Some epidemiologic studies have suggested a relationship between added sugar consumption and increased risk of obesity [25-27]. Other studies have disputed these findings [28,29]. Several recent studies have shown an association between sugar sweetened beverage consumption and increased risk of obesity in children as well as in adults who are genetically predisposed to obesity [30-32].

The American Heart Association (AHA) has recently issued a Scientific Statement suggesting that individuals limit added sugars to no more than half of their discretionary caloric allowance; that is, no more than 150 and $100 \mathrm{cal} /$ day for most American men and women, respectively [9]. The AHA statement acknowledges that these recommendations are based on limited data and that relatively few randomized, prospective trials have been conducted. Many of the studies suggesting links between added sugars and cardiovascular disease are either epidemiologic, which establish associations rather than cause and effect [27,28], or experiments comparing pure fructose versus pure glucose at exaggerated levels, a model system that does not reflect the way that sugars are consumed in the normal human diet [33-35]. Very few studies have explored the effects of sucrose or High Fructose Corn Syrup (HFCS), the major added sugars in the U.S., at normal population consumption levels [36].

It has been argued that the fructose moiety of both sucrose and HFCS may be particularly worrisome due to its effects on body weight and cardiovascular risk factors [37-39]. Both sucrose and HFCS are comprised of roughly 50\% glucose and 50\% fructose. Sucrose is half fructose/half glucose, while the two major forms of HFCS in common use, HFCS-42 and HFCS-55, are comprised of $42 \%$ 
fructose $/ 53 \%$ glucose $/ 5 \%$ glucose oligomers and $55 \%$ fructose $/ 42 \%$ glucose $/ 3 \%$ glucose oligomers, respectively [8].

While consumption of HFCS increased significantly in the U.S. between 1970 and 1999 largely at the expense of the sucrose it replaced, its use has been in steady decline for the past decade [40]. It should be noted that fructose and added sugars from all sources have declined in the United States since 1999 [41,42]. At present, the middle $50 \%$ of the American adult population for consumption of total fructose represents an intake of $10 \%$ of total calories which corresponds to $20 \%$ of total calories from added sugars. However, more sucrose is still consumed in the U.S. each year than HFCS, and worldwide, nine times as much sucrose is used than HFCS [8].

It has been postulated that differences in metabolism between glucose and fructose may be a contributing factor to weight gain and obesity, and increased risk of cardiovascular disease and various metabolic conditions such as the metabolic syndrome and diabetes [25-28]. It has also been postulated that consumption of fructose from any source, whether it be sucrose or HFCS or any of the other glucose/fructose containing nutritive sweeteners, may independently contribute to hypertension, dyslipidemias and insulin resistance [38,39-43].

With this information as background, the current study was undertaken to explore whether two different amounts of sucrose or HFCS consumed at levels equal to the 25 th and 50th percentile of the US adult population consumption level for total fructose have any adverse impact on body weight or body composition and blood lipids or blood pressure in a free living, longitudinal study lasting 10-weeks. Overweight and obese individuals were chosen since they are most at risk for these metabolic abnormalities. To our knowledge, this is the first prospective study to examine the effects of added sugars on overweight/obese individuals when sugars are consumed at levels typical of the adult population.

\section{Experimental Section}

\subsection{Study Design}

This study was a 10-week, randomized, prospective, double blinded trial involving overweight/obese subjects between the ages of 25 and 60 . The consequences were compared of consuming sucrose or HFCS at $10 \%$ or $20 \%$ of total calories (25th or 50th percentile population intake levels of fructose) as a component of the usual diet in a free living environment. The study was approved by the Western Institutional Review Board (Project identification code: 20091302, date of approval: 27 July 2009) and the University of Central Florida Institutional Review Board and all participants signed uniformed consent documents.

\subsection{Subjects}

Men and women between the ages of 25 and 60 years of age with Body Mass Index (BMI) $27.0-35.0 \mathrm{~kg} / \mathrm{m}^{2}$ were recruited. Exclusions included current enrollment in any commercial weight loss program, prescription medicines or supplements for weight loss, or a greater than five pound change in weight during the past three months. Individuals with a history of orthopedic limitations that would interfere with the ability to meet prescribed exercise, a history of heart problems, a history of 
major surgery within the last three months, clinically diagnosed eating disorders or any gastrointestinal disorder, dietary restrictions or allergies to any component of the diet or which would limit the ability to adhere to dietary requirements of the study were all excluded. Users of tobacco products or individuals consuming more than 14 alcoholic beverages per week were also excluded.

Interested individuals were initially screened over the phone to determine eligibility based on self-reported data. Those who were eligible participated in a clinical screening visit to verify a qualifying height and weight, to rule out undiagnosed hypertension, and to obtain a fasting blood sample for the measurement of glucose, insulin, lipids and C Reactive protein (CRP). Individuals who failed to meet study inclusion criteria were dismissed at that time.

Each subject performed a second screening visit one week later. During this visit, research dietitians assessed participant dietary intake by analyzing a completed three day food record using Nutrient Data System Research (NDS-R) Software (University of Minnesota, Minneapolis, MN, USA). Body composition was determined by Dual X-Ray Absorptiometry (General Electric i-DXA, Madison, WI, USA). All females were required to have a negative serum pregnancy test prior to DXA testing. Repeat measurements were performed after the end of 10 weeks. All cholesterol samples were sent to a certified research-based laboratory with error rates of less than $1 \%$.

\subsection{Intervention}

Throughout the 10-week intervention participants were required to incorporate an amount of low-fat (1\% fat) flavored milk sweetened with either HFCS or sucrose (nutritional composition is provided in Table 1), according to their randomly assigned group:

GROUP \#1 (HFCS 10\%): 10\% of recommended calories provided from HFCS in the milk (equivalent to the 25 th percentile for intake of fructose for adults).

GROUP \#2 (HFCS 20\%): 20\% of recommended calories provided from HFCS in the milk (50th percentile for intake of fructose for adults).

GROUP \#3 (Suc 10\%): 10\% of recommended calories from sucrose in the milk (25th percentile intake of fructose for adults).

GROUP \#4 (Suc 20\%): 20\% of recommended calories from sucrose in the milk (50th percentile intake of fructose).

\subsection{Nutritional Plans}

Participants were provided a structured eating plan based on the American Diabetes Association (ADA) exchange lists. Overall caloric level was determined using the Mifflin-St. Jeor calculation for Resting Energy Expenditure (REE) with activity factor included based on responses to a physical activity questionnaire. Daily consumption levels of the test beverage were then calculated according the group assignment (10\% or $20 \%$ calories from added sugar). Increases in added sugar from the milk were accounted for by reductions in the number of other carbohydrate exchanges allowed. As indicated in Table 1, each carton of milk contained either 11 grams of HFCS or sucrose or $22 \mathrm{~g}$ of HFCS or sucrose. The milk was prepared by mixing either HFCS or sucrose solids into a standard $1 \%$ milk solution. The amount of solids added were dependent on whether the milk was intended for those at the $10 \%$ consumption level ( $11 \mathrm{~g}$ of solids per $240 \mathrm{~mL}$ of milk) or the $20 \%$ consumption level 
(22 $\mathrm{g}$ of solids per $240 \mathrm{~mL}$ of milk). There was no fructose or glucose added to the milk so the only that was present were those in the lactose moiety or the added HFCS or sucrose solids.

Individuals in the $10 \%$ HFCS or sucrose groups were given milk containing the lower amount of HFCS or sucrose, while individuals in the $20 \%$ HFCS or sucrose groups were given milk containing the higher amount of HFCS or sucrose containing milk. Then, based on the calculated caloric needs of each participant, they were assigned a number of cartons that provided them with either $10 \%$ or $20 \%$ of their estimated caloric needs from the sweeteners. This could have been as few as two cartons or many as five or six cartons.

Table 1. Nutrient composition of milk.

\begin{tabular}{cccc}
\hline Formulation & \multicolumn{3}{c}{$\mathbf{1 \%}$ Fat Milk and Carbohydrate } \\
\hline Ingredient & $\begin{array}{c}\text { 1\% Low Fat } \\
\text { Milk }\end{array}$ & $\begin{array}{c}\text { HFCS or } \\
\text { Sucrose } \\
\text { Solids }\end{array}$ & $\begin{array}{c}\text { HFCS or } \\
\text { Sucrose Solids }\end{array}$ \\
\hline Consumption Level & $240 \mathrm{~mL}$ & $10 \%$ & $20 \%$ \\
Dose & 2.5 & 0 & $22 \mathrm{~g}$ \\
Fat (g) & 13 & 11 & 0 \\
Carbohydrate (g) & 8 & 0 & 22 \\
Protein (g) & 110 & & 0 \\
Calories & & 44 & 88 \\
From Milk & 110 & 154 & 198 \\
\hline From Added Sweetener & & &
\end{tabular}

HFCS: High Fructose Corn Syrup.

Both participants and investigators were blinded to the type of sugar given to each participant. However, while participants were also blinded to whether they were in a $10 \%$ or $20 \%$ sugar group, investigators were aware of this information since it was necessary to prescribe the right number of other carbohydrate exchanges. Compliance with diet and test beverage was checked weekly based on completed checklists turned in during a visit in which participants would also have their weight measured and be supplied with another weeks supply of test beverage. Participants were withdrawn if they failed to consume the prescribed milk for five consecutive days or if they failed to maintain an overall level of consumption of at least $80 \%$ of that prescribed. All sweeteners were supplied in $1 \%$, low-fat milk (Tetra Pak, Denton, TX, USA) as described above.

\subsection{Data Analysis}

Data were checked for normalcy and analyzed using a two way (time and group assignment) Analysis of Variance with repeated measures. Significant time X group assignment interactions were probed by assessing the within subject change within each of the 4 groups separately. In addition, changes over the course of 10-weeks (week 10-baseline) were calculated and among group differences assessed by one way Analysis of variance (ANOVA). For all analyses the alpha value was set at 0.05. All data were analyzed using SPSS Advanced Statistics V18 (SPSS ${ }^{\circledR}$ Statistics is a comprehensive system for analyzing data, (IBM, Armonk, NY, USA). 


\section{Results}

\subsection{Compliance and Participant Attrition}

Of the 86 individuals who started the study, 65 completed the 10-week intervention. Reasons for attrition were varied and included unwillingness to maintain milk consumption levels, inability to attend clinical appointments and investigator determined exclusion due to non-compliance. Only data from the 65 participants who completed the study are presented herein. Baseline characteristics are shown in Table 2. Compliance with prescribed milk intake was very high with over $96 \%$ of recommended servings being consumed. Milk consumption among finishers was ranged from two to six eight ounce cups/day in order to achieve either $10 \%$ or $20 \%$ of calories from HFCS or sucrose.

\subsection{Dietary Intake}

Despite the addition of the sugar-sweetened milk, participants followed a structured dietary plan designed to provide a weight-maintenance level of calories. However, as shown in Table 3, according to analysis of 3 day food diaries caloric intake increased $(p<0.001)$, irrespective of group assignment (interaction $p=0.925$ ). There were also increases in carbohydrate and protein and total sugar intake (all $p<0.001$ ). Of these, only sugar intake was affected by group assignment (interaction $<0.01$ ). While there was a trend towards a larger increase in consumption for both total and added sugar in the $20 \%$ groups compared to the $10 \%$ groups statistical significance was only reached for the comparisons between HFCS $20 \%$ and sucrose $10 \%$ for total sugar, and HFCS $20 \%$ vs. both $10 \%$ groups for added sugar. No changes were observed in intake of dietary fat $(p>0.05)$.

\subsection{Body Composition}

Changes in body mass and measures of adiposity and body composition are presented in Table 4. While there were no significant changes in body weight for any individual group, a slight increase in body weight was observed when assessing the entire cohort (mean $=2.2 \pm 4.9 \mathrm{lbs}, p<0.01$ ). While this was statistically significant, during the screening of prospective participants being "weight stable" was defined as there being no change in weight greater than 5 lbs during the past 30 days. Such a change in weight as observed in the present study would therefore still be classified as being "weight-stable" according the criteria used for screening participants. This observed increase in weight represented an increase in percent body fat and fat mass $(p<0.01$ for both). Importantly changes in these measures were comparable across the 4 groups suggesting group assignment did not influence the degree of weight or fat gain. 
Table 2. Baseline participant characteristics.

\begin{tabular}{|c|c|c|c|c|c|}
\hline \multirow[t]{2}{*}{ Participants } & $\begin{array}{c}\text { All } \\
(n=65, M=34, \\
\text { F }=31)\end{array}$ & $\begin{array}{c}\text { HFCS } 10 \% \\
(n=17, M=9, \\
F=8)\end{array}$ & $\begin{array}{c}\text { HFCS 20\% } \\
\begin{array}{c}(n=17, M=8, \\
F=9)\end{array}\end{array}$ & $\begin{array}{c}\text { Sucrose } 10 \% \\
(n=18, M=12, \\
F=6)\end{array}$ & $\begin{array}{c}\text { Sucrose } 20 \% \\
(n=13, M=5, \\
F=8)\end{array}$ \\
\hline & Mean \pm S.D & Mean \pm S.D & Mean \pm S.D & Mean \pm S.D & Mean \pm S.D \\
\hline Age (years) & $39.12 \pm 11.76$ & $39.82 \pm 11.60$ & $39.33 \pm 10.94$ & $41.15 \pm 12.24$ & $36.48 \pm 12.50$ \\
\hline \multicolumn{6}{|l|}{ Gender $(\%)$} \\
\hline Female & 52 & 53 & 47 & 67 & 38 \\
\hline Male & 48 & 47 & 53 & 33 & 62 \\
\hline \multirow[t]{2}{*}{ Race $(\%)$} & Caucasian $=68$ & Caucasian $=71$ & Caucasian $=68$ & Caucasian $=72$ & Caucasian $=69$ \\
\hline & $\begin{array}{c}\text { African American }=9 \\
\begin{array}{c}\text { Hispanic }=14 \\
\text { Asian }=6 \\
\text { Other }=3\end{array}\end{array}$ & $\begin{array}{c}\text { African American }=12 \\
\text { Hispanic }=12 \\
\text { Other }=6\end{array}$ & $\begin{array}{c}\text { African American }=12 \\
\text { Hispanic }=18 \\
\text { Asian }=12\end{array}$ & $\begin{array}{c}\text { African American }=11 \\
\text { Hispanic }=6 \\
\text { Asian }=11\end{array}$ & $\begin{array}{c}\text { African American }=23 \\
\text { Other }=8\end{array}$ \\
\hline Height (inches) & $66.37 \pm 3.77$ & $66.71 \pm 4.79$ & $66.30 \pm 3.66$ & $66.74 \pm 4.05$ & $65.37 \pm 3.71$ \\
\hline Body Weight (lbs) & $179.55 \pm 36.12$ & $180.66 \pm 40.98$ & $180.31 \pm 38.36$ & 178.30 & $178.87 \pm 33.37$ \\
\hline BMI & $28.58 \pm 4.31$ & $27.79 \pm 4.30$ & $29.43 \pm 3.51$ & $27.96 \pm 3.97$ & $29.30 \pm 3.88$ \\
\hline Waist Circumference $(\mathrm{cm})$ & $89.51 \pm 11.71$ & $89.16 \pm 12.63$ & $91.04 \pm 12.75$ & $88.67 \pm 11.64$ & $89.18 \pm 10.39$ \\
\hline Body Fat $\%$ & $36.31 \pm 8.19$ & $36.12 \pm 7.74$ & $38.63 \pm 7.51$ & $32.75 \pm 8.91$ & $37.79 \pm 7.90$ \\
\hline Systolic Blood Pressure (mmHg) & $112.46 \pm 13.53$ & $111.36 \pm 12.81$ & $112.10 \pm 14.21$ & $116.00 \pm 16.58$ & $110.76 \pm 10.81$ \\
\hline Diastolic Blood Pressure (mmHg) & $73.60 \pm 11.55$ & $70.50 \pm 15.61$ & $73.48 \pm 10.87$ & $75.15 \pm 10.99$ & $75.35 \pm 7.50$ \\
\hline Cholesterol (mg/dL) & $183.60 \pm 36.61$ & $196.77 \pm 39.53$ & $178.24 \pm 30.99$ & $175.00 \pm 38.25$ & $183.39 \pm 34.42$ \\
\hline Triglycerides (mg/dL) & $128.60 \pm 73.61$ & $122.05 \pm 57.34$ & $126.48 \pm 69.99$ & $121.05 \pm 67.09$ & $143.39 \pm 95.55$ \\
\hline High Density Lipoprotein (mg/dL) & $51.13 \pm 14.36$ & $53.09 \pm 13.40$ & $48.24 \pm 15.63$ & $52.70 \pm 15.81$ & $50.57 \pm 29.64$ \\
\hline Low Density Lipoprotein (mg/dL) & $106.95 \pm 32.19$ & $118.64 \pm 37.71$ & $104.67 \pm 23.76$ & $98.10 \pm 34.68$ & $105.56 \pm 29.64$ \\
\hline
\end{tabular}

M: male; F: female. 
Table 3. Changes in macronutrient profile of the diet after ten weeks of sugar sweetened milk consumption as part of a eucaloric diet.

\begin{tabular}{|c|c|c|c|c|c|c|}
\hline Variable & Time & HFCS $10 \%$ & HFCS $20 \%$ & Suc $10 \%$ & Suc $20 \%$ & All \\
\hline Energy Intake & Baseline & $2204 \pm 1076$ & $2115 \pm 477$ & $2542 \pm 1407$ & $2086 \pm 964$ & $2260 \pm 1048$ \\
\hline (kcal) & Week 10 & $2588 \pm 758$ & $2546 \pm 836$ & $2970 \pm 1786$ & $2328 \pm 686$ & $2644 \pm 1160 * * *$ \\
\hline \multicolumn{7}{|c|}{ Interaction $p=0.925$} \\
\hline Carbohydrate & Baseline & $273.6 \pm 125.1$ & $255.9 \pm 62.6$ & $323.4 \pm 178.2$ & $236.6 \pm 102.3$ & $277.2 \pm 129.2$ \\
\hline (g) & Week 10 & $341.6 \pm 97.5$ & $374.3 \pm 132.2$ & $383.0 \pm 242.2$ & $348.6 \pm 102.0$ & $363.4 \pm 158.8 * * *$ \\
\hline \multicolumn{7}{|c|}{ Interaction $p=0.389$} \\
\hline Fat & Baseline & $83.5 \pm 57.2$ & $82.2 \pm 26.5$ & $94.9 \pm 58.5$ & $90.8 \pm 53.7$ & $87.7 \pm 49.8$ \\
\hline (g) & Week 10 & $86.2 \pm 38.2$ & $72.2 \pm 30.7$ & $99.4 \pm 66.9$ & $62.2 \pm 23.4$ & $82.3 \pm 46.4$ \\
\hline \multicolumn{7}{|c|}{ Interaction $p=0.074$} \\
\hline Protein & Baseline & $89.6 \pm 28.2$ & $91.8 \pm 22.7$ & $99.7 \pm 59.7$ & $85.9 \pm 39.2$ & $92.5 \pm 40.1$ \\
\hline (g) & Week 10 & $122.0 \pm 31.9$ & $109.1 \pm 33.8$ & $144.2 \pm 69.5$ & $108.0 \pm 29.7$ & $122.7 \pm 47.6^{* * *}$ \\
\hline \multicolumn{7}{|c|}{ Interaction $p=0.165$} \\
\hline Total Sugar & Baseline & $126.4 \pm 65.9$ & $110.6 \pm 43.2$ & $154.4 \pm 121.0$ & $108.9 \pm 69.2$ & $127.5 \pm 82.6$ \\
\hline (g) & Week 10 & $199.6 \pm 56.1 * * *$ & $247.9 \pm 99.5 * * *$ & $218.8 \pm 144.6 * *$ & $215.4 \pm 83.6^{* * *}$ & $220.5 \pm 102.7 * * *$ \\
\hline \multicolumn{7}{|c|}{ Interaction $p<0.05$} \\
\hline
\end{tabular}

Note: ** Different within group than baseline, $p<0.01$; *** different within group than baseline, $p<0.001$.

Table 4. Changes in body mass, body composition and waist circumference after ten weeks of sugar sweetened milk consumption as part of a eucaloric diet.

\begin{tabular}{|c|c|c|c|c|c|c|}
\hline Variable & Time & HFCS $10 \%$ & HFCS $20 \%$ & Suc $10 \%$ & Suc $20 \%$ & All \\
\hline Body Mass & Baseline & $185.4 \pm 44.3$ & $184.5 \pm 35.3$ & $179.3 \pm 34.4$ & $182.2 \pm 39.9$ & $182.8 \pm 37.7$ \\
\hline (lbs) & Week 10 & $186.2 \pm 43.9$ & $187.9 \pm 36.0$ & $181.4 \pm 33.9$ & $184.4 \pm 41.1$ & $185.0 \pm 37.9 * *$ \\
\hline \multicolumn{7}{|c|}{ Interaction $p=0.507$} \\
\hline Waist & Baseline & $90.4 \pm 13.5$ & $90.9 \pm 14.0$ & $88.6 \pm 11.2$ & $90.2 \pm 11.6$ & $90.0 \pm 12.4$ \\
\hline$(\mathrm{cm})$ & Week10 & $90.2 \pm 13.2$ & $91.8 \pm 14.2$ & $89.5 \pm 11.1$ & $90.6 \pm 12.1$ & $90.5 \pm 12.4$ \\
\hline \multicolumn{7}{|c|}{ Interaction $p=0.678$} \\
\hline Body Fat & Baseline & $35.6 \pm 7.3$ & $38.7 \pm 7.2$ & $32.6 \pm 9.2$ & $37.7 \pm 4.8$ & $36.0 \pm 7.7$ \\
\hline$(\%)$ & Week 10 & $35.8 \pm 7.3$ & $38.8 \pm 7.0$ & $33.8 \pm 9.1$ & $38.1 \pm 5.0$ & $36.5 \pm 7.5 * *$ \\
\hline \multicolumn{7}{|c|}{ Interaction $p=0.075$} \\
\hline Fat Mass & Baseline & $64.2 \pm 23.5$ & $68.0 \pm 15.5$ & $57.3 \pm 23.0$ & $66.4 \pm 16.2$ & $63.7 \pm 20.1$ \\
\hline (lbs) & Week 10 & $64.8 \pm 23.0$ & $69.5 \pm 15.9$ & $59.7 \pm 22.6$ & $68.2 \pm 17.7$ & $65.3 \pm 20.1 * *$ \\
\hline \multicolumn{7}{|c|}{ Interaction $p=0.532$} \\
\hline Fat Free Mass & Baseline & $120.3 \pm 27.0$ & $115.3 \pm 28.5$ & $121.7 \pm 21.7$ & $114.3 \pm 26.3$ & $118.1 \pm 26.0$ \\
\hline (lbs) & Week 10 & $120.9 \pm 29.3$ & $116.9=28.1$ & $120.8 \pm 22.0$ & $117.3 \pm 27.9$ & $118.6 \pm 26.3$ \\
\hline \multicolumn{7}{|c|}{ Interaction $p=0.080$} \\
\hline
\end{tabular}

\subsection{Blood Pressure and Blood Lipids}

No changes in systolic $(114.12 \pm 12.71$ vs. $113.92 \pm 11.52 \mathrm{mmHg}, p>0.05)$ or diastolic blood pressure $(73.91 \pm 12.01$ vs. $75.38 \pm 9.11 \mathrm{mmHg}, p>0.05)$ were observed, and these parameters were unaffected by the treatment group (interactions $p=0.792$ and $p=0.666$, respectively). 
Blood lipid data are presented in Table 5. In summary, sweetened milk consumption was seen to result in an overall decrease in HDL $(p<0.01)$ and increase in total cholesterol to HDL ratio $(p<0.01)$, which were unaffected by treatment group (interaction $p>0.05$ ). However, changes in total cholesterol, LDL and Apo B were different among the groups - change in HFCS 20\% was more positive than that observed in Sucrose 20\% However, this was largely driven by an unexpected decrease in this group for these three measures. Triglycerides were unchanged after the 10 -weeks $(p>0.05)$.

Table 5. Changes in blood lipids after ten weeks of sugar sweetened milk consumption as part of a eucaloric diet.

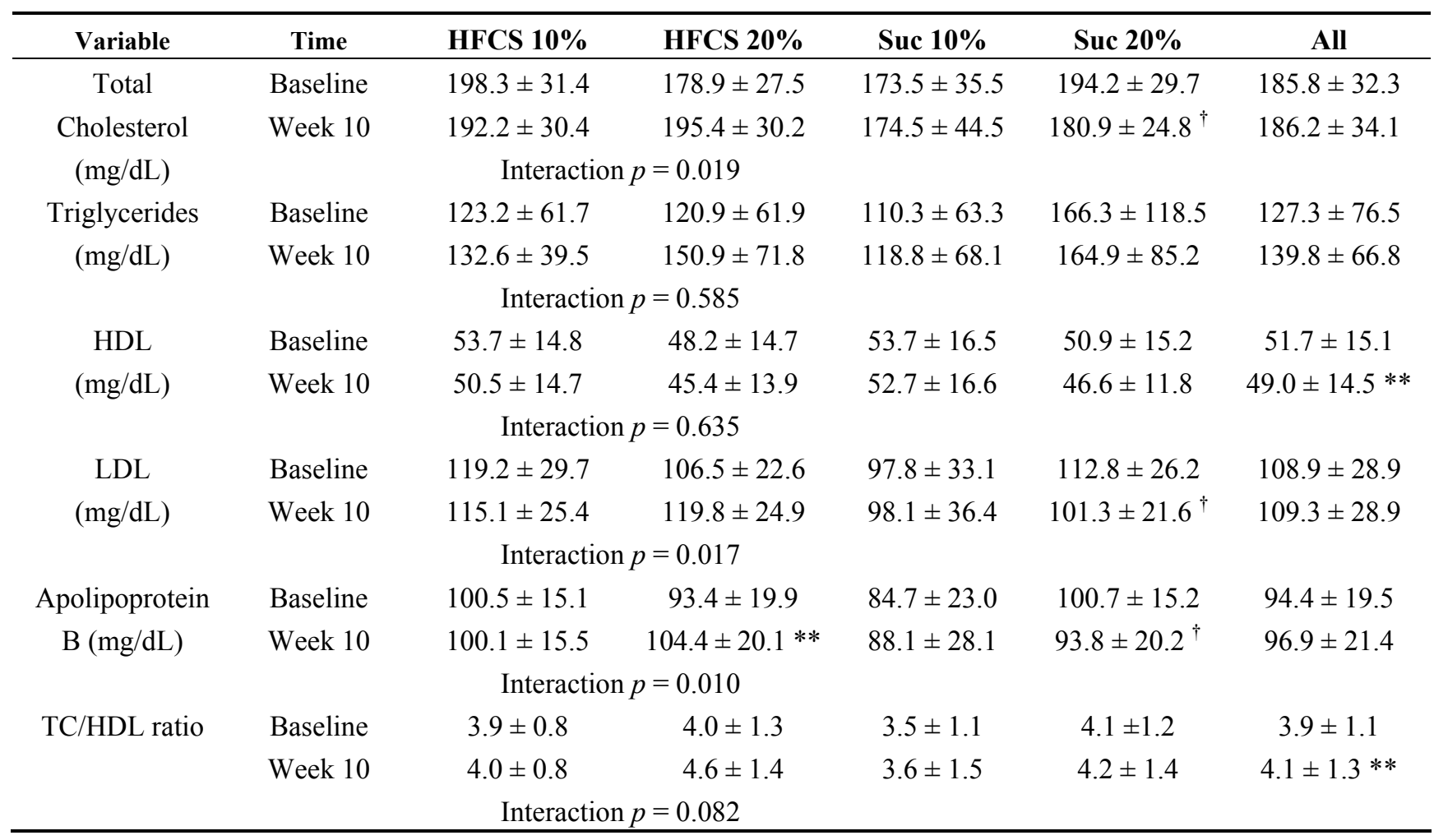

Note: $* *$ different within group than baseline, $p<0.01{ }^{\dagger}$ Change (Week 10-baseline) different than HFCS $20 \%(p<0.05)$.

\section{Discussion}

This double blind study examined changes in weight and body composition, blood lipids and blood pressure before and after a 10 -week, free living intervention during which low fat (1\%) milk was consumed, sweetened with either sucrose or HFCS to deliver $10 \%$ or $20 \%$ of calories from the sweetener in the context of mixed nutrient meals. Our main findings are: (1) when consumed as part of a eucaloric (weight-stable) diet for a 10-week period, fructose containing sugars do not promote weight gain or an atherogenic lipid profile even when consumed at two to four times the level currently recommended by the AHA [9]; and (2) there are no differences between sucrose and HFCS in these measures at typical levels of sweetener consumption.

Sugars are ubiquitous in the food supply and have been classified as either "naturally occurring" or "added" [9,41,42]. Based on loss-adjusted food availability data from the U.S. Department of Agriculture Economic Research Service [44], the energy contribution from sugars and sweeteners increased by $57 \mathrm{cal} /$ day between 1970 and 2008, while total calories increased $515 \mathrm{cal} /$ day; it should 
be noted that added fats and cereals/starches increased 231 and $193 \mathrm{cal} /$ day, respectively [44]. The two leading sources of fructose in the American diet are sucrose and HFCS. Worldwide, individuals consume nine times as much fructose in the form of sucrose as is consumed in the form of HFCS [8].

In the current study, baseline sugar consumption for the cohort was slightly above population norms (baseline sugar consumption $=127.5 \pm 82.6 \mathrm{~g} /$ day). Total sugar consumption increased by approximately 60 grams/day in both the 10\% HFCS and 10\% sucrose and by over 100 grams per day in the HFCS 20\% arm and sucrose 20\% arm. As already indicated, individuals followed an overall American Diabetes Association Exchange Diet. They were counseled to substitute the sweetened milk for other carbohydrate exchanges within this diet. When increases in added sugars were assessed it is apparent that individuals in the HFSC and sucrose $10 \%$ arm were more able to substitute the sweetened milk for other carbohydrates in their diets when compared with the HFCS and sucrose $20 \%$ groups.

While we cannot be certain that the target of $10 \%$ or $20 \%$ total energy as sucrose or HFCS was attained during the intervention, these increases, coupled with the high level of adherence observed on daily and weekly dietary checklists, suggests that the targets were largely met.

Randomized clinical feeding trials have shown inconsistent results from testing the effects of added sugars on weight gain [27,28,45-49]. Differences in populations studied, study design, study instruments and methods may have contributed to these inconsistencies. Studies utilizing a model of pure fructose versus pure glucose have demonstrated differences in their effects on short term energy regulating hormones such as insulin, leptin and ghrelin [33-35]. It has been argued that differences in metabolism between fructose and glucose in the liver could contribute to increased appetite and calories consumed following fructose consumption thereby potentially stimulating weight gain and obesity [33-35]. It should be emphasized that neither fructose nor glucose is consumed in isolation to any appreciable degree. Studies which have compared the normally consumed sugars of HFCS and sucrose have not found differences in energy regulating hormones [50,51]. In the current study, no significant changes in body weight, body fat or waist circumference were found in any individual group following a 10 -week, free living experiment where either $10 \%$ or $20 \%$ of calories consumed came from added sugars in the form of sucrose or HFCS.

While the mean weight gain for the entire cohort of $2.2 \mathrm{lbs}$ was technically within the "weight stable" range as defined by this study, it nonetheless bears further scrutiny since it suggests that individuals may have had difficulty incorporating $10 \%$ or $20 \%$ of calories from added sugars into their normal diets. The average weight gain of $2.2 \mathrm{lbs}$. over the 10-week study is consistent with the average daily increase of $384 \mathrm{kcals}$ when all four groups were combined. This weight gain is also consistent with results other investigators have reported when adding $25 \%$ of calories as either fructose or glucose in free living 10 week studies [33].

Some evidence suggests that increased intake of added sugars may contribute to elevated blood pressure [10-12]. These studies include animal trials where rodents were fed extraordinary doses of fructose (greater than $60 \%$ of energy), acute ingestion studies where humans were fed high doses of different sugars (25\% of energy as fructose or glucose) [33-35], and epidemiologic studies such as the Framingham Heart Study [52] and the Nurses Health Trial [53], where consumption of one or more sugar sweetened soft drink per day increased the odds of developing high blood pressure. Results of human studies, however, are inconsistent and very few data are available from randomized, 
prospective trials at normal population consumed levels of added sugars. A recent meta-analysis of controlled feeding trials found that isocaloric substitution of fructose for other carbohydrates in humans did not adversely affect blood pressure [54].

Lipid abnormalities such as elevation of fasting plasma triglycerides have been demonstrated in diets high in sucrose and fructose (greater than $20 \%$ of energy) $[55,56]$. Fasting triglyceride elevations from sucrose or fructose may be more marked in men than women [18], sedentary or obese individuals [57], and in those with the metabolic syndrome [58]. Recent systematic reviews and meta-analyses, however, have reported that when fructose is substituted isocalorically for other carbohydrates neither fasting [59] or postprandial [60] triglycerides rise. Findings are inconsistent on the effects of added sugars on LDL-cholesterol levels [53-55].

In the current study, there was an overall decrease in HDL $(p<0.01)$ and increase in total cholesterol to HDL ratio $(p<0.01)$, however these measures were unaffected by treatment group (interaction $p>0.05$ ). Triglycerides rose approximately $10 \%$ which was not significant. These changes are all consistent with a diet that replaces dietary fat with carbohydrates, particularly simple sugars. Diets such as the Dietary Approaches to Stop Hypertension (DASH) Diet that replace fat with carbohydrate from fruits, vegetables, whole grains and non-fat and low-fat dairy products that lower HDL cholesterol somewhat but do not increase triglycerides [17,61]. It is also possible that the failure to observe an increase in plasma triglycerides in the overall cohort was somewhat dependent on the slightly elevated baseline sugar consumption, however, as already noted, total sugar consumption rose appropriately in all four groups.

The observed response in triglycerides in HFCS $20 \%$ was not a surprising observation. Increased intake of carbohydrates has been shown to promote formation of nascent very-low-density lipoprotein (VLDL) particles by combining glycerol, free fatty acids and Apo B, thus increasing plasma triglycerides Although our study was not designed the explore the hepatic synthesis of VLDL the increased Apo B observed in this group supports our speculation.

It should also be noted that the sugars were delivered in $1 \%$ milk and as a result total protein intake also increased. This may have altered the food intake and also hepatic lipid metabolism. Thus, our reported results, related to lipid parameters must be treated with some caution.

In the current study there were no changes in either systolic or diastolic blood pressure $(p>0.05)$ and these parameters were not affected by treatment group. These findings are different from some studies that have suggested that added sugars may raise blood pressure. Moreover, some epidemiologic studies such as the Framingham Heart Study have shown that consumption of $\geq 1$ sugar sweetened carbonated soft drink per day increases the risk of developing high blood pressure [52]. Other studies have not demonstrated elevated blood pressure from increased sugar intake. Thus, the effects of intakes of simple sugars, particularly at average levels on blood pressure remain uncertain.

A strength of the current study is that it is a double blind, randomized, prospective study with a relatively large sample size which explored normal population consumed levels of fructose as delivered through the normally consumed sweeteners, sucrose or HFCS. A further strength of this study is that it examined overweight and obese subjects who are more prone to the metabolic abnormalities assessed in the current investigation. Weaknesses of the current study include that subjects were only followed for 10 weeks and that children, adolescents and individuals over the age of 60 were excluded. 
Further studies employing larger numbers of subjects, different population groups (e.g., adolescents and individuals over the age of 60) may be warranted.

\section{Conclusions}

In summary, consumption of added sugars, either from sucrose or HFCS at $10 \%$ and $20 \%$ of calories consumed (normal population consumption levels) in this 10 week trial, showed no significant changes in any group with respect to weight, adiposity, or abdominal adiposity, and no adverse effects on triglycerides, LDL, or blood pressure in a randomized, controlled trial of free living individuals lasting 10-week. These findings are consistent with other recent studies showing metabolic similarities between HFCS and sucrose [51,62-69].

Although no individual group gained significant weight, the slight weight gain when considering the entire cohort and the mixed response to lipid parameters, such as HDL and total cholesterol, warrant further trials of longer duration and larger number of subjects.

\section{Availability of Supporting Data}

The data set supporting the results of this article is included within the article.

\section{Acknowledgments}

JL participated overall, day to day supervision of the protocol. DK delivered nutritional portions of the protocol. SP delivered nutritional portions of the protocol. VN delivered nutritional portions of the protocol. KM helped conceptualize the protocol. ZY was responsible for some of the laboratory testing and delivered nutritional portions of the protocol. BL was responsible for scheduling patients, performing some testing and coordinating appointments. JR was responsible for conceptualizing the protocol and had overall supervision of the protocol. All authors participated in the writing of the manuscript and have approved it.

This research was made possible through an unrestricted grant from the Corn Refiners Association.

\section{Conflicts of Interest}

JR's laboratory has received unrestricted grant funding to conduct research trials and JR has received consulting fees from a variety of companies, organizations, publishers or trade associations that utilize, market or publish information about fructose, high fructose corn syrup, or sucrose and hence, have an ongoing interest in the metabolism and health effects of these sugars. The other study authors report no competing interests.

\section{References}

1. Flegal, K.M.; Carroll, M.D.; Ogden, C.L.; Curtin, L.R. Prevalence and trends in obesity among US adults, 1999-2008. JAMA 2010, 303, 235-247.

2. Hedley, A.A.; Odgen, C.L.; Johnson, C.L.; Carroll, M.D.; Curtin, L.R. Prevalence of overweight and obesity among US children, adolescents, and adults, 1999-2002. JAMA 2004, 291, 2847-2850. 
3. Dietz, W.H.; Robinson, T.N. Overweight children and adolescents. N. Engl. J. Med. 2005, 325, 2100-2109.

4. Poirier, P.; Giles, T.D.; Bray, G.A.; Hong, Y.; Stern, J.S.; Pi-Sunyer, F.X.; Eckel, R.H. Obesity and cardiovascular disease: Pathophysiology, evaluation, and effect of weight loss: An update of the 1997 American Heart Association scientific statement on obesity and heart disease from the obesity committee of the council on nutrition, physical activity, and metabolism. Circulation 2006, 113, 898-918.

5. Eckel, R. Obesity and heart disease: A statement for the healthcare professionals from the Nutrition Committee, American Heart Association. Circulation 1997, 96, 3248-3250.

6. Sigman-Grant, M.; Morita, J. Defining and interpreting intakes of sugars. Am. J. Clin. Nutr. 2003, $78,815 \mathrm{~S}-826 \mathrm{~S}$.

7. Hein, G.L.; Storey, M.L.; White, J.S.; Lineback, D.R. Highs and lows of high fructose corn syrup. Nutr. Today 2005, 40, 253-256.

8. White, J. Straight talk about high-fructose corn syrup: What it is and what it ain't. Am. J. Clin. Nutr. 2008, 88, 1716S-1721S.

9. Johnson, R.; Appel, L.; Brands, M.; Howard, B.; Lefevre, M.; Lustig, R.; Sacks, F.; Steffen, L.; Wylie-Rosett, J.; American Heart Association Nutrition Committee of the Council on Nutrition, Physical Activity, and Metabolism and the Council on Epidemiology and Prevention. Dietary sugars intake and cardiovascular health: A scientific statement from the American Heart Association. Circulation 2009, 120, 1011-1020.

10. Feig, D.I.; Soletsky, B.; Johnson, R.J. Effect of allopurinol on blood pressure of adolescents with newly diagnosed essential hypertension: A randomized trial. JAMA 2008, 300, 924-932.

11. Nguyen, S.; Choi, H.K.; Lustig, R.H.; Hsu, C.Y. Sugar-sweetened beverages, serum uric acid, and blood pressure in adolescents. J. Pediatr. 2009, 154, 807-813.

12. Bremer, A.A.; Auinger, P.; Byrd, R.S. Relationship between insulin resistance-associated metabolic parameters and anthropometric measurements with sugar-sweetened beverage intake and physical activity levels in US adolescents: Findings from the 1999-2004 National Health and Nutrition Examination Survey. Arch. Pediatr. Adolesc. Med. 2009, 163, 328-335.

13. Van der Schaaf, M.R.; Koomans, H.A.; Joles, J.A. Dietary sucrose does not increase twenty-four-hour ambulatory blood pressure in patients with either essential hypertension or polycystic kidney disease. J. Hypertens. 1999, 17, 453-454.

14. Hellerstein, M.K. Carbohydrate-induced hypertriglyceridemia: Modifying factors and implications for cardiovascular risk. Curr. Opin. Lipidol. 2002, 13, 33-40.

15. Mensink, R.P.; Zock, P.L.; Kester, A.D.; Katan, M.B. Effects of dietary fatty acids and carbohydrates on the ratio of serum total to HDL cholesterol and on serum lipids and apolipoproteins: A meta-analysis of 60 controlled trials. Am. J. Clin. Nutr. 2003, 77, 1146-1155.

16. Appel, L.J.; Sacks, F.M.; Carey, V.J.; Obarzanek, E.; Swain, J.F.; Miller, E.R.; Conlin, P.R.; Erlinger, T.P.; Rosner. B.A.; Laranjo, N.M.; et al. Effects of protein, monounsaturated fat, and carbohydrate intake on blood pressure and serum lipids: Results of the OmniHeart randomized trial. JAMA 2005, 294, 455-2464. 
17. Howard, B.V.; Van Horn, L.; Hsia, J.; Manson, J.E.; Stefanick, M.L.; Wassertheil-Smoller, S.; Kuller, L.H.; LaCroix, A.Z.; Langer, R.D.; Lasser, N.L. Low-fat dietary pattern and risk of cardiovascular disease: The Women's Health Initiative Randomized Controlled Dietary Modification Trial. JAMA 2006, 295, 655-666.

18. Bantle, J.P.; Raatz, S.K.; Thomas, W.; Georgopoulos, A. Effects of dietary fructose of plasma lipids in healthy subjects. Am. J. Clin. Nutr. 2000, 72, 1128-1134.

19. Black, R.N.; Spence, M.; McMahon, R.O.; Cuskelly, G.J.; Ennis, C.N.; McCance, D.R.; Young, I.S.; Bell, P.M.; Hunter, S.J. Effect of eucaloric high-and low-sucrose diets with identical macronutrient profile on insulin resistance and vascular risk: A randomized controlled trial. Diabetes 2006, 55, 3566-3572.

20. Marckmann, P.; Raben, A.; Astrup, A. Ad libitum intake of low-fat diets rich in either starchy foods or sucrose: Effects on blood lipids, factor VII coagulant activity, and fibrinogen. Metabolism 2000, 49, 731-735.

21. Liu, S.; Manson, J.E.; Buring, J.E.; Stampfer, M.J.; Willett, W.C.; Ridker, P.M. Relation between a diet with a high glycemic load and plasma concentrations of high-sensitivity C-reactive protein in middle-aged women. Am. J. Clin. Nutr. 2002, 75, 492-498.

22. Price, K.D.; Price, C.S.; Reynolds, R.D. Hyperglycemia-induced ascorbic acid deficiency promotes endothelial dysfunction and the development of atherosclerosis. Atherosclerosis 2001, $158,1-12$.

23. Ceriello, A.; Bortolotti, N.; Crescentini, A.; Motz, E.; Lizzio, S.; Russo, A.; Ezsol, Z.; Tonutti, L.; Taboga, C. Antioxidant defenses are reduced during the oral glucose tolerance test in normal and non-insulin-dependent diabetic subjects. Eur. J. Clin. Investig. 1998, 28, 329-333.

24. Ma, S.W.; Tomlinson, B.; Benzie, I.F. A study of the effect of oral glucose loading on plasma oxidant: Antioxidant balance in normal subjects. Eur. J. Nutr. 2005, 44, 250-254.

25. Schulze, M.; Manson, J.; Ludwig, D.; Colditz, G.; Stampfer, M.; Willett, W.; Hu, F. Sugar-sweetened beverages, weight gain, and incidence of type 2 diabetes in young and middle-aged women. JAMA 2004, 292, 927-934.

26. Bachman, C.M.; Baranowski, T.; Nicklas, T.A. Is there an association between sweetened beverages and adiposity? Nutr. Rev. 2006, 64, 153-174.

27. Malik, V.S.; Schulze, M.B.; Hu, F.B. Intake of sugar-sweetened beverages and weight gain: A systematic review. Am. J. Clin. Nutr. 2006, 84, 274-288.

28. Johnson, L.; Mander, A.P.; Jones, L.R.; Emmett, P.M.; Jebb, S.A. Is sugar-sweetened beverage consumption associated with increased fatness in children? Nutrition 2007, 23, 557-563.

29. Forshee, R.A.; Anderson, P.A.; Storey, M.L. Sugar-sweetened beverages and body mass index in children and adolescents: A meta-analysis. Am. J. Clin. Nutr. 2009, 89, 441-442.

30. Ebbeling, C.B.; Feldman, H.A.; Chomitz, V.R.; Antonelli, T.A.; Gortmaker, S.L.; Osganian, S.K.; Ludwig, D.S. A randomized trial of sugar-sweetened beverages and adolescent body weight. N. Engl. J. Med. 2012, 367, 1407-1416.

31. De Ruyter, J.C.; Olthof, M.R.; Seidell, J.C.; Katan, M.A. Trial of sugar-free or sugar-sweetened beverages and body weight in children. N. Engl. J. Med. 2012, 367,1397-1406. 
32. Qi, Q.; Chur, A.Y.; Kang, J.H.; Jensen, M.K.; Curhan, G.C.; Pasquale, L.R.; Ridker, P.M.; Hunter, D.J.; Willett, W.C.; Rimm, E.B.; et al. Sugar-sweetened beverages and genetic risk of obesity. N. Engl. J. Med. 2012, 367, 1387-1396.

33. Stanhope, K.; Schwarz, J.; Keim, N.; Griffen, S.; Bremer, A.; Graham, J.; Hatcher, B.; Cox, C.; Dyachenko, A.; Zhang, W.; et al. Consuming fructose-sweetened, not glucose-sweetened, beverages increases visceral adiposity and lipids and decreases insulin sensitivity in overweight/obese humans. J. Clin. Investig. 2009, 119, 1322-1334.

34. Teff, K.; Elliott, S.; Tschop, M.; Kieffer, T.; Rader, D.; Heiman, M.; Townsend, R.; Keim, N.; D'Alessio, D.; Havel, P. Dietary fructose reduces circulating insulin and leptin, attenuates postprandial suppression of ghrelin, and increases triglycerides in women. JCEM 2004, 89, 2963-2972.

35. Teff, K.L.; Grudziak, J.; Townsend, R.R.; Dunn, T.N.; Grant, R.W.; Adams, S.H. Endocrine and metabolic effects of consuming fructose-and glucose-sweetened beverages with meals in obese men and women: Influence of insulin resistance on plasma triglyceride responses. JCEM 2009, $94,1562-1569$.

36. Lowndes, J.; Kawiecki, D.; Pardo, S.; Nguyen, V.; Melanson, K.; Yu, Z.; Rippe, J. The effects of four hypocaloric diets containing different levels of sucrose or high fructose corn syrup on weight loss and related parameters. Nutr. J. 2012, 11, 55-64.

37. Bray, G.A.; Nielsen, S.J.; Popkin, B.M. Consumption of high fructose corn syrup in beverages may play a role in the epidemic of obesity. Am. J. Clin. Nutr. 2004, 79, 537-543.

38. Johnson, R.; Segal, M.; Sautin, Y.; Nakagawa, T.; Feig, D.; Kang, D.; Gersch, M.; Benner, S.; Sanchez-Lozada, L. Potential role of sugar (fructose) in the epidemic of hypertension, obesity and the metabolic syndrome, diabetes, kidney disease, and cardiovascular disease. Am. J. Clin. Nutr. 2007, 86, 899-906.

39. Bray, G.A. Fructose: Should we worry? Int. J. Obes. 2008, 32, S127-S131.

40. Fereday, M.; Forber, G.; Girardello, S.; Midgley, C.; Nutt, T.; Powell, N.; Todd, M. HFCS Industry Annual Review-A Year of Changing Expectations. Sweetener Analysis; LMC International Ltd.: Oxford, UK, 2007; pp. 1-8.

41. Marriott, B.P.; Cole, N.; Lee, E. National Estimates of dietary fructose intake increased from 1977 to 2004 in the United States. J. Nutr. 2009, 139, 1228S-1235S.

42. Marriott, B. Worldwide Consumption of Sweeteners and Recent Trends. In Fructose, High Fructose Corn Syrup, Sucrose and Health; Rippe, J.M., Ed.; Springer: New York, NY, USA, 2014; pp. 87-111.

43. Bray, G. Fructose: Pure, white, and deadly? Fructose, by any other name is a health hazard. J. Diabetes Sci. Technol. 2010, 4, 1003-1007.

44. Wells, H.F.; Buzby, J.C. Dietary Assessment of Major Trends in US Food Consumption, 1970-2005; USDA-Economic Research Service: Washington, DC, USA, 2008.

45. Ebbeling, C.B.; Feldman, H.A.; Osganian, S.K.; Chomitz, V.R.; Ellenbogen, S.J.; Ludwig, D.S. Effects of decreasing sugar-sweetened beverage consumption on body weight in adolescents: A randomized, controlled pilot study. Pediatrics 2006, 117, 673-680. 
46. Maersk, M.; Belza, A.; Stødkilde-Jørgensen, H.; Ringgaard, S.; Chabanova, E.; Thomsen, H.; Pedersen, S.B.; Astrup, A.; Richelsen, B. Sucrose-sweetened beverages increase fat storage in the liver, muscle, and visceral fat depot: A 6-mo randomized intervention study. Am. J. Clin. Nutr. 2012, 95, 283-289.

47. Lowndes, J.; Kawiecki, D.; Angelopoulos, T.; Rippe, J. Fructose containing sugars do not cause changes in weight, body composition or abdominal fat when consumed as part of a eucaloric (weight-stable) diet. Obesity 2010, 18, S51.

48. Sievenpiper, J.L.; de Souza, R.J.; Mirrahimi, A.; Yu, M.E.; Carleton, A.J.; Beyene, J.; Chiavaroli, L.; Di Buono, M.; Jenkins, A.L.; Leiter, L.A.; et al. Effect of fructose on body weight in controlled feeding trials: A systematic review and meta-analysis. Ann. Intern. Med. 2012, 156, 291-304.

49. Wang, D.D.; Sievenpiper, J.L.; de Souza, R.J.; Chiavaroli, L.; Ha, V.; Cozma, A.I.; Mirrahimi, A.; Yu, M.E.; Carleton, A.J.; di Buono, M.; et al. The effects of fructose intake on serum uric acid vary among controlled dietary trials. J. Nutr. 2012, 142, 916-923.

50. Melanson, K.J.; Zukley, L.; Lowndes, J.; Nguyen, V.; Angelopoulos, T.J.; Rippe, J.M. Effects of high-fructose corn syrup and sucrose consumption on circulating glucose, insulin, leptin, and ghrelin and on appetite in normal-weight women. Nutrition 2007, 23, 103-112.

51. Soenen, S.; Westerterp-Plantenga, M.S. No differences in satiety or energy intake after high-fructose corn syrup, sucrose, or milk preloads. Am. J. Clin. Nutr. 2007, 86, 1586-1594.

52. Dhingra, R.; Sullivan, L.; Jacques, P.F.; Wang, T.J.; Fox, C.S.; Meigs, J.B.; D’Agostino, R.B.; Gaziano, J.M.; Vasan, R.S. Soft drink consumption and risk of developing cardiometabolic risk factors and the metabolic syndrome in middle-aged adults in the community. Circulation 2007, $116,480-488$.

53. Fung, T.T.; Malik, V.; Rexrode, K.M.; Manson, J.E.; Willett, W.C.; Hu, F.B. Sweetened beverage consumption and risk of coronary heart disease in women. Am. J. Clin. Nutr. 2009, 89, 1037-1042.

54. Ha, V.; Sievenpiper, J.; de Souza, R.J.; Chiavaroli, L.; Wang, D.D.; Cozma, A.I.; Mirrahimi, A.; Yu, M.E.; Carleton, A.J.; Dibuono, M.; et al. Effect of fructose on blood pressure: A systematic review and meta-analysis of controlled feeding trials. Hypertension 2012, 59, 787-795.

55. Lê, K.A.; Tappy, L. Metabolic effects of fructose. Curr. Opin. Clin. Nutr. Metab. Care 2006, 9, 469-475.

56. Parks, E.J.; Hellerstein, M.K. Carbohydrate-induced hypertriacylglycerolemia: Historical perspective and review of biological mechanisms. Am. J. Clin. Nutr. 2000, 71, 412-433.

57. Fried, S.K.; Rao, S.P. Sugars, hypertriglyceridemia, and cardiovascular disease. Am. J. Clin. Nutr. 2003, 78, 873S-880S.

58. Miller, M.; Stone, N.J.; Ballantyne, C.; Bittner, V.; Criqui, M.; Ginsberg, H.; Goldberg, A.C.; Howard, W.J.; Jacobson, M.S.; Kris-Etherton, P.; et al. Triglycerides and cardiovascular disease. A scientific statement from the american heart association. Circulation 2011, 123, 2292-2333.

59. Chiavaroli, L.; Mirrahimi, A.; de Souza, R.J.; Cozma, A.I.; Ha, V.; Wang, D.D.; Yu, M.E.; Carleton, A.J.; Beyene, J.; Kendall, C.W.C.; et al. Does fructose consumption elicit a dose-response effect on fasting triglycerides? A systematic review and meta-regression of controlled feeding trials. Can. J. Diabetes 2012, 36, S37. 
60. Sievenpiper, J.L.; Wang, D.D.; de Souza, R.J.; Cozma, A.I.; Ha, V.; Chiavaroli, L.; Mirrahimi, A.; Carleton, A.J.; Beyene, J.; Kendall, C.W.C.; et al. Effect of fructose on postprandial triglycerides: A systematic review and meta-analysis of controlled feeding trials. Atherosclerosis 2014, 232, 125-133.

61. Obazanek, E.; Sacks, P.M.; Vollmer, W.M.; Bray, G.A.; Miller, E.R., III; Lin, P.H.; Karanja, N.M.; Most-Windhauser, M.M.; Moore, T.J.; Swain, J.F.; et al. Effects on blood lipids of a blood pressure-lowering diet: The Dietary Approaches to Stop Hypertension (STOP) Trial. Am. J. Clin. Nutr. 2001, 74, 80-89.

62. Akhavan, T.; Anderson, G.H. Effects of glucose-to-fructose ratios in solutions on subjective satiety, food intake, and satiety, food intake, and satiety hormones in young men. Am. J. Clin. Nutr. 2007, 86, 1354-1363.

63. Le, M.T.; Frye, R.F.; Rivard, C.J.; Cheng, J.; McFann, K.K.; Segal, M.S.; Johnson, R.J.; Johnson, J.A. Effects of high-fructose corn syrup and sucrose on the pharmacokinetics of fructose and acute metabolic and hemodynamic responses in healthy subjects. Metabolism 2012, 61, 641-651.

64. Rippe, J.; Angelopoulos, T. Sucrose, high fructose corn syrup and fructose, and their potential health effects: What do we really know? Adv. Nutr. 2013, 4, 236-245.

65. Bravo, S.; Lowndes, J.; Sinnett, S.; Yu, Z.; Rippe, J. Consumption of sucrose and high-fructose corn syrup does not increase liver fat or ectopic fat deposition in muscles. Appl. Physiol. Nutr. Metab. 2013, 38, 681-688.

66. Yu, Z.; Lowndes, J.; Rippe, J. High-fructose corn syrup and sucrose have equivalent effects on energy-regulating hormones at normal human consumption levels. Nutr. Res. 2013, 33, 1043-1052.

67. Rippe, J.M. The metabolic and endocrine response and health implications of consuming sugar-sweetened beverages: Findings from recent randomized controlled trials. Adv. Nutr. 2013, 4, 677-686.

68. Klurfeld, D.M.; Foreyt, J.; Angelopoulos, T.J.; Rippe, J.M. Lack of evidence for high fructose corn syrup as the cause of the obesity epidemic. Int. J. Obes. 2013, 37, 771-773.

69. White, J.S. Challenging the fructose hypothesis: New perspectives on fructose consumption and metabolism. Adv. Nutr. 2013, 4, 246-256.

(C) 2014 by the authors; licensee MDPI, Basel, Switzerland. This article is an open access article distributed under the terms and conditions of the Creative Commons Attribution license (http://creativecommons.org/licenses/by/3.0/). 